\title{
Numerical modelling of partially filled aircraft fuel tanks submitted to Hydrodynamic Ram
}

\author{
D. Varas, R. Zaera, J. López-Puente* \\ Department of Continuum Mechanics and Structural Analysis, University Carlos III of Madrid, Avda. de la Universidad, 30, 28911 Leganés, Madrid, Spain
}

Keywords:

Hydrodynamic Ram

Fluid-structure interaction

Aircraft vulnerability

Impact

Fuel tank

\begin{abstract}
A B S T R A C T
Hydrodynamic Ram (HRAM) is a phenomenon that occurs when a high-kinetic energy object penetrates a fluid-filled container. The projectile transfers its momentum and kinetic energy through the fluid to the surrounding structure, increasing the risk of catastrophic failure and excessive structural damage. This is of particular concern in the design of wing fuel tanks for aircraft since it has been identified as one of the important factors in aircraft vulnerability. Usually the HRAM phenomenon is analyzed considering completely filled tanks, but its effect on partially filled containers should also be taken into account due to the fact that tanks use to be impacted under these conditions. In the present paper, the commercial finite element code LS-DYNA has been used to simulate an HRAM event created by a steel spherical projectile impacting a partially water-filled aluminium square tube. The ALE formulation is employed to reproduce the event. Experimental tests which indicate the pressure at different points of the fluid, displacement of the walls and cavity evolution for different impact velocities, are compared with the numerical results in order to assess the validity and accuracy of the ALE technique in reproducing such a complex phenomenon.
\end{abstract}

\section{Introduction}

The process by which a high-speed projectile penetrates a fluidfilled tank and transfers kinetic energy to the surrounding walls is known as Hydrodynamic Ram (HRAM). The HRAM effect in fuel tanks is identified as one of the important factors in aircraft vulnerability since the fuel tanks represent the largest exposed area of all the vulnerable components. HRAM is especially dangerous for aircraft with extremely lightweight designs, since the structural resistance of their integral fuel tanks cannot be improved by strengthening the airframe because it would counteract the requirements of a lightweight design.

Vulnerability to HRAM has been usually related to military aircraft, but commercial airplanes are not exempt of its effect. In 1990 the Federal Aviation Administration (FAA) established the Aircraft Catastrophic Failure Prevention Research Program, in which the analysis of the effects of an uncontained turbine engine fragment penetrating aircraft fuel tanks [36] was one of the research areas. In 2000, when a Concorde crashed after takeoff from Charles de Gaulle airport (France), the final investigation report revealed that the HRAM had played a significant role in the aircraft failure.

Hydrodynamic Ram can be divided into four principal stages: shock, drag, cavitation and exit (Fig. 1). Each stage contributes

\footnotetext{
* Corresponding author. Fax: +34 916249430.

E-mail address: jlpuente@ing.uc3m.es (J. López-Puente).
}

to structural damage in a different way and to a different extent. When the projectile penetrates the wall of the fluid-filled structure, it creates damage in the vicinity of the impact point; in addition the impact energy is transferred to the fluid, generating a high-pressure hemispherical shock wave. During the drag phase, the projectile travels through the fluid, while its kinetic energy is partially transformed into fluid motion as the projectile is slowed by fluid drag forces. The displacement of the fluid from the projectile path generates a radial pressure field. In contrast to the pressure field that develops during the shock phase, the fluid is accelerated gradually instead of impulsively. This causes less intense peak pressures but of greater temporal extent. The displacement of fluid during the drag stage forms a cavity behind the projectile. The subsequent expansion and collapse (oscillations) of the cavity is known as the cavitation stage. The oscillations of the cavity can cause significant pressure pulses. The final stage occurs when the projectile exits the container. In contrast to the perforation of the front wall, the exit of the projectile occurs through a pre-stressed wall, caused by the initial shock stage and the subsequent loading by the fluid.

Simulation of HRAM events has been attempted, with more or less success, for over 30 years. The first methods employed to simulate HRAM were based on the use of the Piston Theory for the fluid-structure interaction. This theory assumes the normal reflection of pressure waves when reaching the walls of the structure, resulting in a one-dimensional response mechanism. The Piston Theory was incorporated into two structural analysis codes in or- 


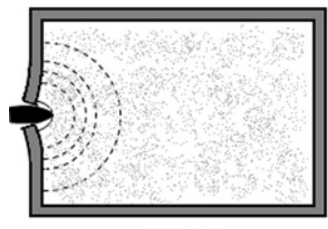

SHOCK

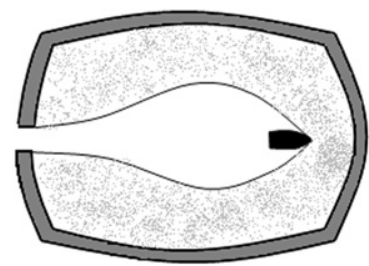

CAVITATION

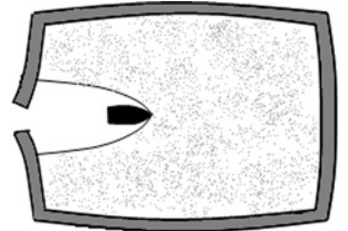

DRAG

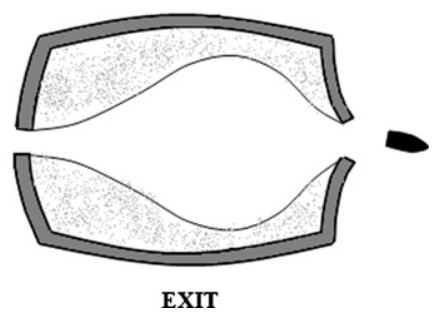

Fig. 1. Phases of Hydrodynamic Ram. der to simulate the HRAM phenomenon [4,2]. The comparison of the numerical results, provided by these two codes, with experimental data $[3,11,6]$ showed a complete lack of agreement and proved the failure of the Piston Theory for Hydrodynamic Ram pressure loading.

Once the Piston Theory was discarded to predict HRAM fluidstructure interaction, Lundstrom [28] proposed the Variable Image Method. Lundstrom described the flow field in terms of a potential function $\phi$ which satisfied the wave equation. The model attempts to approximate the effect of the projectile and cavity by a line of sources distributed along the trajectory behind the projectile. This allows determining the incident pressure wave magnitude by means of the Bernoulli's equation and then uses it to calculate the pressure wave reflected from the structures' walls [29]. In general, this methodology has not been particularly successful and the geometries that can be analyzed are limited. However, the Variable Image Method solved some of the planar assumptions in the Piston Theory model, and provided a more realistic coupling between the fluid and the structure. Other researchers [47] developed a plastic deformation model to predict the structural response of fuel cell walls due to HRAM in contrast to other works in which only the elastic regime was considered. The model requires the knowledge of the total impulse imparted by the fluid to the fuel cell.

Other codes such as HRSR (Hydraulic Ram Structural Response) [15], ERAM or EHRSR were developed [10], but all of them showed their limitations and lack of accuracy on predicting the consequences of an HRAM event since none of them fully coupled the mechanisms of fluid-structure interaction, nor did they allow for a complex, engineered structure. The complicated physics and mechanics of HRAM phenomena were not satisfactory solved until higher-order numerical algorithms were incorporated into the codes in the late 1980's.

Coupled Euler-Lagrange methods have been under development since the early-to-mid 1990's. They combine the desirable characteristics of Lagrangian and Eulerian formulations. These methods are used in multiple industries for a wide variety of analysis in which fluids interact with structures or when high distortions may appear [36,1,37,16,38,9,7,27,21], including airbag and tire-water dynamics in the automobile field [30,22], the impact of bird strikes on aircraft [14,17], and the effects of sloshing on ships [50]. The Arbitrary Lagrangian Eulerian (ALE) technique is widely used in those kind of problems. It can be viewed as a hybrid between the Lagrangian and Eulerian methods which combines the best characteristics of both. It avoids the mesh distortion problem of the Lagrangian formulation and allows to precisely define the contours unlike in the Eulerian formulation.
In the last few years, there have been new advances in development and use of computational methods for fluid-structure interactions due to the interest of reach more effective computational techniques $[5,42,46,20,41,25]$ and solve more difficult problems motivated by different industries, such as aeronautics, naval or more recently biomedical sciences.

As an example of the increasing interest on solving industrial fluid-structure problems, it is worth to mention the recent works of Petitpas et al. [32] and Lecysyn et al. [23,24] in which a ballistic impact on an industrial tank, filled with a toxic fluid and made of steel, is studied. The authors propose an analytical model to reproduce the behaviour of the projectile on the fluid and study its influence on the toxic liquid ejection and the droplets generated. Interesting results are obtained in those works.

The modelling of coupled problems of fluid-structure interaction such as HRAM, has been proven to be a complicated task and is still quite challenging. This is of great importance since analytical solutions can provide only a limited understanding of the nature of the behaviour. In addition, the HRAM phenomenon has usually been studied in completely filled containers without taking into account its effect on the structure on a partially filled tank. Nevertheless, it is more probable that an aircraft fuel tank could be impacted when it is not completely filled because they are fully filled for a short period of time.

The authors of this work have previously observed the different behaviour between completely and partially filled tanks impacted by a projectile [43]. The partially filled tubes show some particularities due to the presence of air above the fluid inside the tank. This fact makes that the cavity inside the tank raises a layer of fluid that impacts the wall. This impact affects the structure in a different way than in a completely filled tank. In addition, the distribution of the fluid inside the tank influences the wall deformations. The authors also showed [44] the capabilities of different numerical techniques for the simulation of the HRAM phenomenon for completely fluid filled tanks.

In the present paper, numerical simulations of a partially waterfilled aluminium square tube subjected to impact by steel spherical projectiles at different velocities are shown. The simulations are performed with the software LS-DYNA applying the Arbitrary Lagrangian Eulerian (ALE) technique for the fluid. Experimental tests providing the pressure in different points of the fluid, deformation of the walls and cavity evolution for different impact velocities are compared with the numerical results. The ALE technique was already proved to be valid on simulating the HRAM phenomenon on fluid filled tanks [44], however it would be necessary to confirm that it also reproduces the particular characteristics observed in the partially filled cases. Under these conditions two different fluids are present (water and air), and hence the water suffers much great displacements.

\section{General description of the ALE technique}

Lagrangian methods are mostly used for problems in which deformation is not extremely large. The mesh moves in space with the material, so that tracking surfaces and applying boundary conditions can be done easily. If the solids suffer large deformations and mesh distortion is high, the calculation will stop and the nodes of the mesh will need to be repositioned in order to continue the calculation. This process is necessary every time the mesh becomes too distorted for the calculation to continue. Therefore, obtaining solutions to the fluid dynamics aspect of a high velocity impact in a fluid-structure interaction event (as HRAM phenomenon) is not reasonable using a purely Lagrangian method due to the high deformation that the fluid mesh suffers.

On the other hand, purely Eulerian descriptions are typically used for fluid calculations. The mesh remains fixed in space while 
the material passes through it. Surfaces and boundary conditions are difficult to track, however the mesh distortion is not a problem because the mesh is not distorted. Therefore, obtaining solutions to the structural dynamics aspects of a high velocity impact in a fluid-structure interaction event is not feasible using a purely Eulerian method due to the deformation that the structural mesh suffers. The ALE formulation contains both pure Lagrangian and pure Eulerian formulations being able to address both the fluid and structural dynamics of an event.

The ALE approach is based on the arbitrary movement of a reference domain which, additionally to the common material domain and spatial domain, is introduced as a third domain [39]. The problem is formulated in this reference domain, which later will correspond to the finite element mesh. The arbitrary movement of the reference frame enables to deal with moving boundaries, free surfaces and large deformations, and interface contact problems. In the ALE description of LS-DYNA [26], an arbitrary referential coordinate is introduced in addition to the Lagrangian and Eulerian coordinates. The ALE equations are derived by substituting the relationship between the material time derivative and the reference configuration time derivative:

$$
\begin{aligned}
\frac{\partial f\left(X_{i}, t\right)}{\partial t} & =\frac{\partial f\left(x_{i}, t\right)}{\partial t}+\left(v_{i}-u_{i}\right) \frac{\partial f\left(x_{i}, t\right)}{\partial x_{i}} \\
& =\frac{\partial f\left(x_{i}, t\right)}{\partial t}+\omega_{i} \frac{\partial f\left(x_{i}, t\right)}{\partial x_{i}}
\end{aligned}
$$

where $X_{i}$ is the Lagrangian coordinate, $i$ the referential coordinate, $x_{i}$ the Eulerian coordinate, and $v_{i}$ and $u_{i}$ are the material and the mesh velocities, respectively. In order to simplify the equations, the convective velocity term $\omega_{i}=v_{i}-u_{i}$ is introduced.

Thus, the governing equations for the ALE formulation are given by:

- The conservation of mass equation.

$$
\frac{\partial \rho}{\partial t}=-\rho \frac{\partial v}{\partial x_{i}}-\omega_{i} \frac{\partial \rho}{\partial x_{i}}
$$

- The conservation of momentum equation.

$$
\rho \frac{\partial v_{i}}{\partial t}+\rho \omega_{i} \frac{\partial v_{i}}{\partial x_{j}}=\sigma_{i j, j}+\rho b_{i}
$$

- The conservation of total energy equation.

$$
\rho \frac{\partial E}{\partial t}+\rho \omega_{j} \frac{\partial E}{\partial x_{j}}=\sigma_{i j} v_{i, j}+b_{j} v_{j}
$$

where $\rho$ is density, $\sigma_{i j}$ the stress tensor, $b_{i}$ is body force and $E$ is energy.

Note that the Lagrangian and Eulerian formulations for the conservation equations are obtained setting the mesh velocity equal to the particle velocity, $u=v$, or setting the mesh velocity equal to zero, $u=0$, respectively.

In the LS-DYNA code, the ALE algorithm is implemented using the Split Operator Technique [13] which is a two step process. The ALE method begins working as a Lagrangian finite element code in which the motions of the fluid and the structure are determined and the finite element grid is deformed following the material. As long as the mesh distortion is acceptable, the Lagrangian calculation continues. If it becomes highly distorted, the second step (the advection step) is performed. In the ALE methodology the distorted mesh can be partially restored to its original shape based on predefined criteria for element deformation. Following a prescribed measure of permissible distortion in the element, the element shape is changed; and mass, momentum and energy are fluxed across the old element boundaries to calculate their new values for the new element shape. In general, the ALE methodology permits flow of material across element boundaries preserving a balance for the physical velocity between grid motion and flow [10].

\section{Experimental set-up}

In order to achieve an appropriate and wide validation of the numerical modelling, it is necessary to have enough experimental data. As there is not much available data on pressure, tank walls deformation or cavity evolution of the HRAM phenomenon in the literature, experimental tests were made by the same workers and their results were presented in a previous paper [43]. The tests consisted of high velocity impacts of steel spheres into tanks filled at different levels. Water was selected as fluid for safety reasons and because of its similar density compared to that of kerosene. The sketch of the experimental device used for impact tests is shown in Fig. 2.

The test boxes consisted of 6063-T5 square aluminium tubes $750 \mathrm{~mm}$ long, $150 \mathrm{~mm}$ wide and $2.5 \mathrm{~mm}$ thick. The specimens were closed with two PMMA windows $30 \mathrm{~mm}$ thick, fixed to the specimen with four steel bars; these transparent panels allow the recording of the impact process by means of a Photron Ultima APX-RS digital high-speed camera. A similar set-up was proposed by Nishida et al. [31]. To obtain optimal images of the penetration process, it is necessary an appropriate lighting, which was provided by an Arrisun 12 Plus lamphead with a 1200 W Hydrargyrum Medium-arc Iodide (HMI) lamp. The contact points between PMMA windows and the specimen were sealed with silicone in order to avoid fluid leakage [40].

Two pressure transducers, PCB 138A06 [40], were located at different points to obtain pressure data in the fluid. One of the transducers was placed near the impact point, PTn, and the other was placed far from the impact point, PTf. Two holes were made on the lower wall of the specimen to place both sensors inside the water. Their positions are shown in Fig. 3. A Dewetron DEWE-800 data-acquisition device was used to record the signals. This system can record at a sampling rate of $1 \mu$ s and synchronize the data with the video recording.

A one-stage light gas gun with a 4.7 litre chamber, which stores gas at a maximum pressure of 300 bar, was used with helium. The length of the barrel was $4.5 \mathrm{~m}$ and its calibre $25 \mathrm{~mm}$. The gun was aimed at an armoured steel specimen chamber box $1 \times 1 \times$ $1 \mathrm{~m}^{3}$ inside of which the specimen was placed during the impact test. The chamber had a small circular window in the front for the projectile to pass through, and two large lateral windows to illuminate the specimen and capture the video sequence of the impact, Fig. 2.

The projectile launched against the box was a steel sphere with a diameter of $12.5 \mathrm{~mm}$ and a mass of $8 \mathrm{~g}$. Two impact velocities were chosen: 600 and $900 \mathrm{~m} / \mathrm{s}$. The test boxes were filled with water at different volume fractions: 60,75 and $100 \%$. In this paper, only conditions corresponding to partially filled tanks (60 and 75\%) will be considered for the simulations.

\section{Numerical analysis}

The numerical model was developed with the commercial finite element code LS-DYNA v.971 [26]. This software is particularly suitable for nonlinear dynamic problems, such as impacts or explosions. It also allows the employment of different techniques such as ALE or SPH to solve fluid and fluid-structure problems. A comparison and validation of both techniques to simulate the HRAM phenomenon in a completely filled tube was carried out by the same workers [44]. In order to reproduce the HRAM phenomenon in a partially filled tank, the ALE technique was adopted to model the fluid inside the tank. 


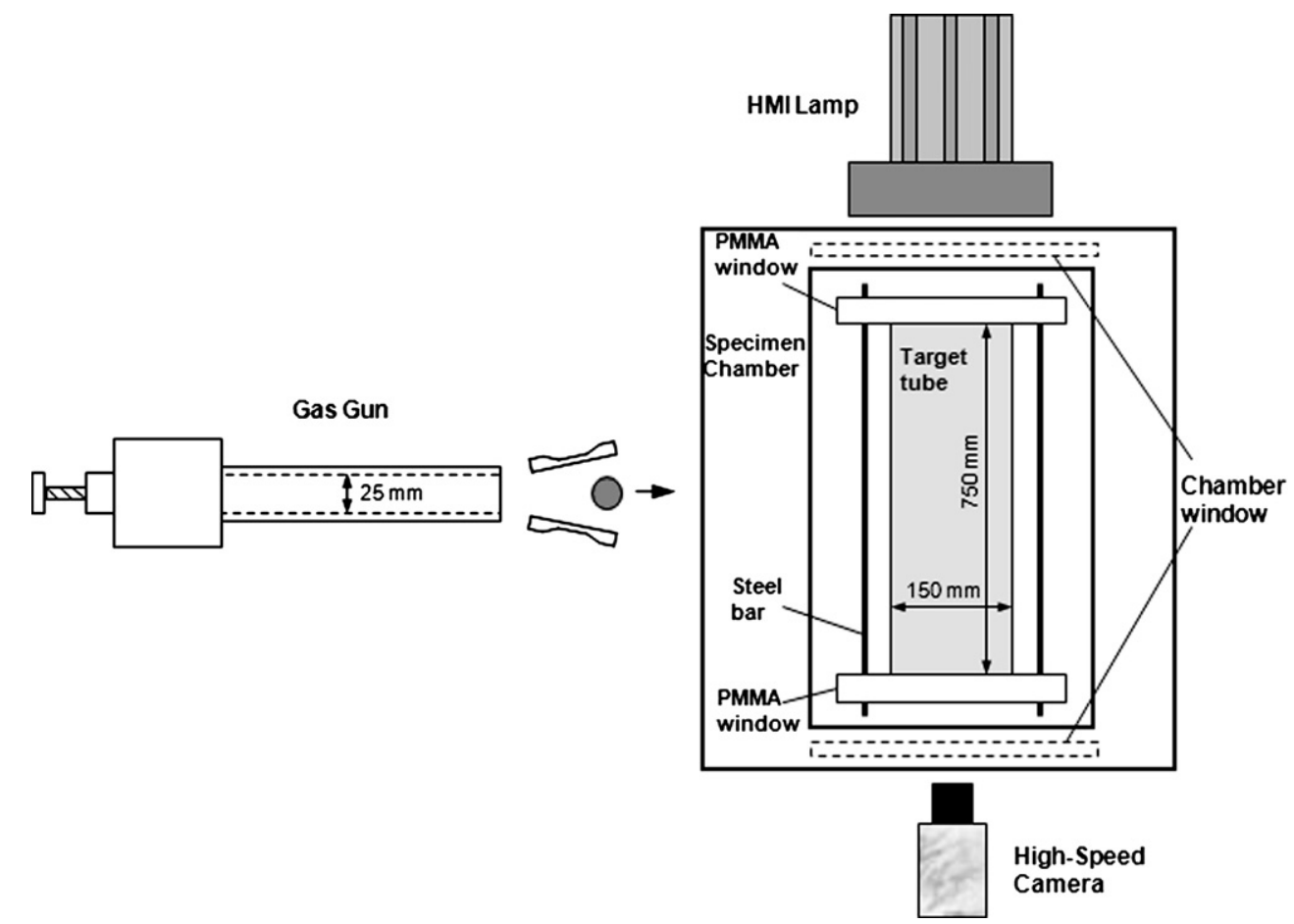

Fig. 2. Sketch of the experimental device used for the impact tests.

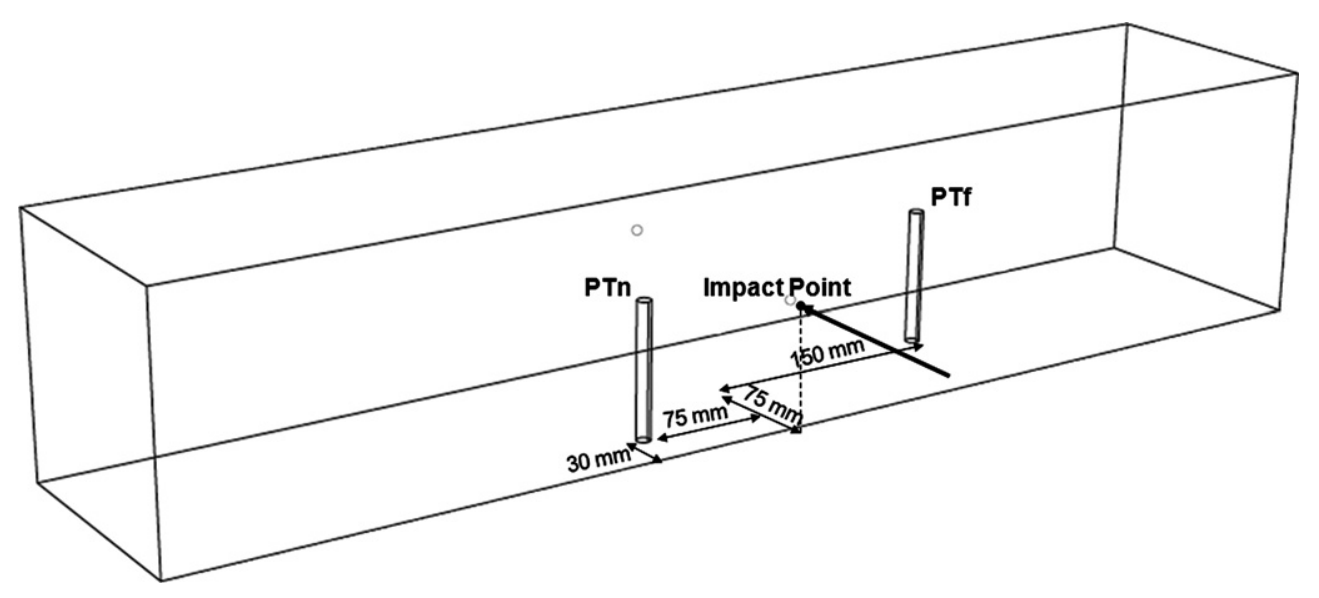

Fig. 3. Sketch of the test box instrumented.

\subsection{Box and projectile FE model}

The symmetry of the problem under consideration allowed modelling half of the whole geometry (Fig. 4, left). Since the nature of this simulation demands a very high mesh density, such a reduction in the model size is very desirable. If the tube would be completely filled with water, the symmetry would allow modelling only a quarter of the whole. The box has been divided in three parts, the walls impacted by the projectile (entry and exit walls), the side walls and the PMMA window.

The impacted walls and the PMMA window were discretized by means of eight-node solid hexahedron Lagrangian elements with reduced integration. A refined mesh, corresponding to the impact zone, and a progressively coarser mesh as the distance to the hit point grows, can be observed in Fig. 4, right. The impacted walls present 5 elements through the thickness and elements of $1 \mathrm{~mm}$ in size in the other two directions near the impacted zone. Based on previous simulations, the mesh size was considered appropriate to reproduce the behaviour of the solids in the impacted zone. Fournode Belytschko-Tsay shell elements were used to discretize the side walls in order to reduce the number of elements. Finally, the mesh of the box consisted of 31804 elements.

The Johnson-Cook hardening relation [18] was selected to model the aluminium of the box. There are more sophisticated hardening relations, such as the Zerilli-Armstrong [49] or the Rusinek-Klepaczko [34,35], but Johnson and Cook's is probably the most widely used among those accounting for equivalent plastic strain $\bar{\varepsilon}^{p}$, equivalent plastic strain rate $\dot{\bar{\varepsilon}}^{p}$ and temperature effects $\theta$. Since numerous efforts have been made in the past to determine their parameters for a large number of metallic materials, it has been implemented in many FE explicit codes. The relation is stated through the following multiplicative equation:

$\sigma_{Y}=\left(A+B\left(\bar{\varepsilon}^{p}\right)^{n}\right)\left(1+C \log \left(\frac{\dot{\bar{\varepsilon}}^{p}}{\dot{\bar{\varepsilon}}_{0}}\right)\right)\left(1-\left(\frac{\theta-\theta_{0}}{\theta_{m}-\theta_{0}}\right)^{m}\right)$

$\theta_{0}$ being the reference temperature and $\theta_{m}$ the melting temperature. The required parameters for the Aluminium 6063-T5 were obtained from $[19,48]$. In order to describe the material failure in 

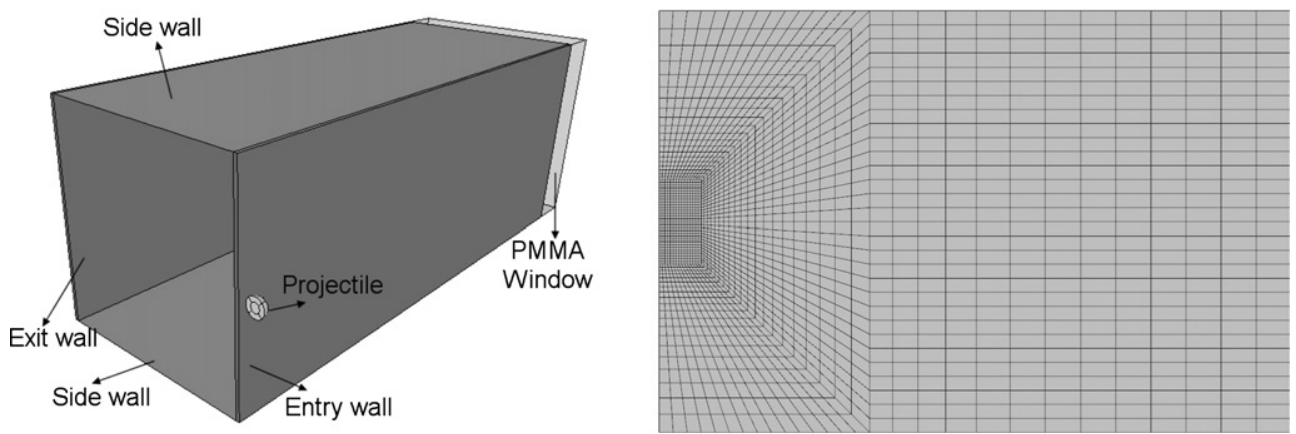

Fig. 4. Left: Box model geometry used for the analysis. Right: Detail of the entry wall mesh.

Table 1

Parameters used in the simulation for the solids.

\begin{tabular}{|c|c|c|c|c|c|c|c|c|c|}
\hline Material & $\rho\left(\mathrm{kg} / \mathrm{m}^{3}\right)$ & $E(\mathrm{GPa})$ & $v$ & $A(\mathrm{MPa})$ & $B(\mathrm{MPa})$ & $n$ & $C$ & $m$ & $D_{1}$ \\
\hline 6063-T5 & 2700 & 71 & 0.33 & 200 & 144 & 0.62 & 0 & 1 & 0.2 \\
\hline Steel & 7830 & 207 & 0.28 & - & - & - & - & - & - \\
\hline PMMA & 1180 & 3 & 0.35 & - & - & - & - & - & - \\
\hline
\end{tabular}

the zone around the impact point, the Johnson-Cook model was used; the relation is described by the following equation:

$$
\begin{aligned}
\varepsilon_{f}= & \left(D_{1}+D_{2} \exp \left(D_{3} \frac{\sigma_{h}}{\bar{\sigma}}\right)\right)\left(1+D_{4} \ln \frac{\dot{\bar{\varepsilon}}^{p}}{\dot{\bar{\varepsilon}}_{0}}\right) \\
& \times\left(1+D_{5}\left(\frac{\theta-\theta_{0}}{\theta_{m}-\theta_{0}}\right)\right)
\end{aligned}
$$

where $\bar{\sigma}$ and $\sigma_{h}$ are the equivalent and the hydrostatic stresses, respectively; the failure occurs when the damage parameter $\sum\left(\Delta \bar{\varepsilon}^{p} / \varepsilon_{f}\right)=1$. In this case, the failure model was used without taking into account the dependence on temperature, strain rate and triaxiality, and hence the constants $D_{2}, D_{3}, D_{4}$ and $D_{5}$ are set to zero. Then, failure occurs when $\bar{\varepsilon}^{p}$ reaches the value $D_{1}$.

An elastic material model was used for the PMMA window since it does not suffer inelastic deformations nor damage. The PMMA properties were obtained from [45]. The projectile was discretized by means of eight-node solid hexahedron Lagrangian elements with reduced integration. The elements size of the projectile, although relatively bigger than that of the wall elements in the impact area, allows to solve in a properly way the contacts with the tank walls. The projectile material was modelled as elastic since no plastic deformation was observed in the tests. The material parameters used are shown in Table 1.

\subsection{Model for the fluid}

It is expected that, due to the HRAM phenomenon, the fluid inside the tank undergoes too large deformations to consider a pure Lagrangian description as an appropriate option. For this reason, a multi-material ALE formulation with a second-order accurate advection method has been chosen for the treatment of the fluid. Multi-material means that each element of the mesh has the ability to contain two or more materials, in this case water and air. The ALE formulation allows the motion of the mesh independently of the material flow without distortion problems.

Fluids inside the box (water and air) are discretized by means of eight-node solid hexahedron elements with an ALE formulation (elform $=11$ in LS-DYNA notation). Strictly, the fluids are discretized by means of an Eulerian mesh, but LS-DYNA considers an Eulerian part as a special case of the ALE formulation, where the nodes are fixed. The air surrounding the box was also considered, being modelled using the same elements as in the fluids inside the box. Modelling this air region is essential to allow the water

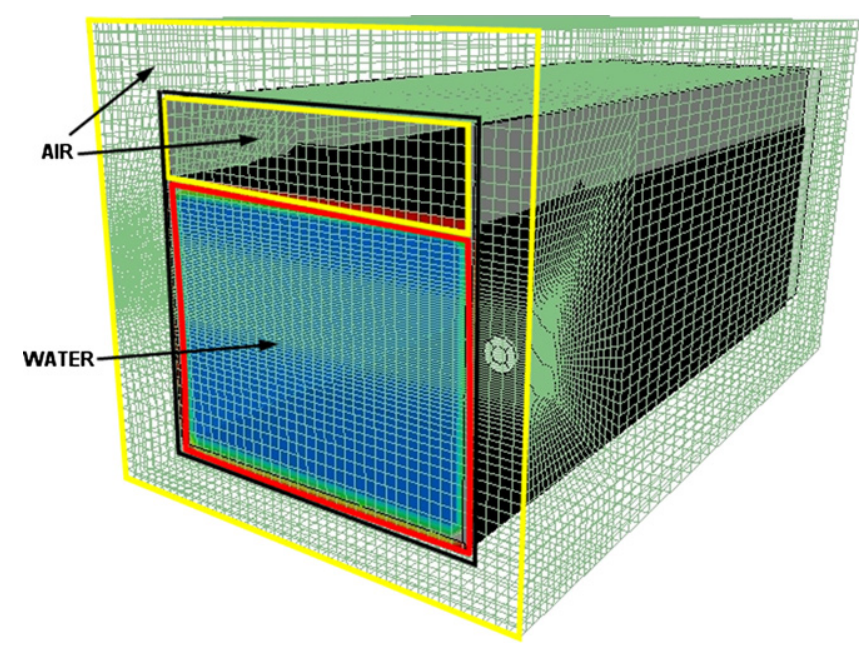

Fig. 5. Mesh of the fluids.

to flow into it, deforming the walls of the structure. To achieve that, water and air meshes have to share the same nodes at their interfaces.

Four discretization densities were analyzed in order to achieve an optimal fluid mesh density; a simplified model without the aluminium tube was used to perform this analysis. The results in terms of projectile deceleration were compared with the classical solution of the movement of a sphere inside a liquid:

$m_{p} \frac{d V_{p}}{d t}=-\frac{1}{2} \rho_{w} A_{0} C_{d} V_{p}^{2}$

where $m_{p}$ and $V_{p}$ denote the bullet mass and velocity, $\rho_{w}$ is the fluid density, $A_{0}$ is the projected frontal area of the projectile and $C_{d}$ is a dimensionless drag coefficient. According to the range of velocities considered, a value of 0.4 for $C_{d}$ was chosen [12]. The optimal mesh density of fluids, determined with this analysis, caused some problems of leakage at the fluid/solid interfaces. To avoid these problems, numerous iterations modifying some of the coupling parameters were made, and also the mesh size was modified in order to match the Lagrangian one at the walls interfaces, obtaining a slightly grosser mesh than the optimal one previously selected. This change in the mesh helped to control the leakage problems. Finally, the fluids inside the box and the surrounding air region present 123038 elements (Fig. 5). 
Table 2

Water and air parameters used in the simulation.

\begin{tabular}{|c|c|c|c|c|c|c|c|c|c|c|}
\hline & $\rho_{0}\left(\frac{\mathrm{kg}}{\mathrm{m}^{3}}\right)$ & $v_{d}(\mathrm{~Pa} \mathrm{~s})$ & $C\left(\frac{\mathrm{m}}{\mathrm{s}}\right)$ & $S_{1}$ & $S_{2}$ & $S_{3}$ & $\gamma_{0}$ & $a$ & $C_{4}$ & $C_{5}$ \\
\hline Water & 1000 & $0.89 \cdot 10^{-3}$ & 1448 & 1.979 & 0 & 0 & 0.11 & 3.0 & - & - \\
\hline Air & 1.22 & $1.77 \cdot 10^{-5}$ & - & - & - & - & - & - & 0.4 & 0.4 \\
\hline
\end{tabular}

The water was modelled using the following viscous constitutive equation (Material Null in LS-DYNA notation)

$\sigma_{i j}=2 v_{d} \dot{\varepsilon}_{i j}^{\prime}-P \delta_{i j}$

in which $\sigma_{i j}$ is the Cauchy stress tensor, $v_{d}$ the dynamic viscosity, $\dot{\varepsilon}_{i j}^{\prime}$ the deviatoric strain rate, and $\delta_{i j}$ the identity tensor. The deviatoric term is negligible compared to the hydrostatic one, both for water and kerosene due to the low value of their dynamic viscosities. The pressure $P$ is calculated as a function of the compression $\mu=\rho / \rho_{0}-1$, where $\rho$ and $\rho_{0}$ are the current and initial densities of the material respectively, and of the internal energy per unit volume $E$, using the Mie-Gruneisen Equation of State based on a cubic shock velocity $\left(u_{s}\right)$-particle velocity $\left(u_{p}\right)$

$P=\frac{\rho_{0} D^{2} \mu\left[1+\left(1-\frac{\gamma_{0}}{2}\right) \mu-\frac{a}{2} \mu^{2}\right]}{\left[1-\left(S_{1}-1\right) \mu-S_{2} \frac{\mu^{2}}{\mu+1}-S_{3} \frac{\mu^{3}}{(\mu+1)^{2}}\right]^{2}}+\left(\gamma_{0}+a \mu\right) E$

for compressed materials and

$P=\rho_{0} D^{2} \mu+\left(\gamma_{0}+a \mu\right) E$

for expanded materials, where $S_{1}, S_{2}$ and $S_{3}$ are coefficients of the slope of the $u_{s}-u_{p}$ curve. $D$ is the intercept of the $u_{s}-u_{p}$ curve, which corresponds to the adiabatic sound speed on water. $\gamma_{0}$ is the Gruneisen gamma and $a$ is the first-order volume correction to $\gamma_{0}$. The required properties and constants of water were obtained from [8].

The air was modelled using the same constitutive equation (Eq. (8)), with the properties of the air, and a Linear Polynomial Equation of State [26]. This equation of state is linear in the internal energy and polynomial in the compression, and defines the pressure $P$ as follows:

$P=C_{0}+C_{1} \mu+C_{2} \mu^{2}+C_{3} \mu^{3}+\left(C_{4}+C_{5} \mu+C_{6} \mu^{2}\right) E$

The air was considered as an ideal gas by setting $C_{0}=C_{1}=C_{2}=$ $C_{3}=C_{6}=0$ and $C_{4}=C_{5}=\gamma-1$, where $\gamma=1.4$ is the ratio of specific heats:

$\gamma=\frac{C_{p}}{C_{v}}$

and the pressure $P$ is given by:

$P=(\gamma-1) \frac{\rho}{\rho_{0}} E$

The properties and parameters used in the simulation for the water and the air are shown in Table 2.

The fluid-structure interaction, for both projectile/fluid and walls/fluid, is achieved by means of a penalty-based ALE-Lagrangian coupling algorithm implemented within LS-DYNA. This allows the fluid material to flow around the structure, but prevents its penetration into the structure mesh applying penalty forces to the fluid and the structure. As soon as a fluid node penetrates the Lagrangian structure, a force of recall is exerted to both the fluid and structural node to make the fluid node return to the surface of the structure avoiding penetration. The penalty forces are proportional to the penetration depth and penalty stiffness, behaving like a spring system.

\section{Results}

In the following section, numerical results of partially filled tubes (75\% and 60\%) impacted at velocities of 900 and $600 \mathrm{~m} / \mathrm{s}$ are analyzed and compared with the available experimental data.

\subsection{HRAM stages}

Firstly, it has been proved that the ALE model employed is capable of reproduce qualitatively an HRAM phenomenon in partially filled tubes, as it was demonstrated in a completely filled case [44]. Each of the HRAM stages have been analyzed in order to prove that they are well represented in partially filled tubes simulations.

- Shock phase. When the projectile penetrates the wall of the fluid-filled structure, the impact energy is transferred to the fluid generating a high-pressure hemispherical shock wave, Fig. 6(a).

- Drag phase. In the drag phase, part of projectile's kinetic energy is transformed into fluid motion. The displacement of the fluid from the projectile path generates a radial pressure field and a cavity behind it. Fig. 6(b) depicts the cavity formed in the simulations.

- Cavitation phase. The expansion and collapse (oscillations) of the cavity is known as the cavitation stage. In the model, the expansion of the cavity is well represented, Fig. 6(c). The collapse is not captured due to the long time needed for the cavity to close.

- Exit phase. The projectile exits the tank through a pre-stressed wall, caused by the initial shock stage and the subsequent loading by the fluid. This is well depicted in Fig. 6(d), where contours of effective stress are shown in the exit wall before being penetrated by the projectile.

\subsection{Time history of the projectile trajectory}

By means of the digital high-speed camera, it is possible to determine the velocity and the position of the projectile inside the fluid. This experimental data is compared with the numerical and analytical results [43] (Figs. 7 and 8), these last obtained from Eq. (7).

The figures show a good correlation between numerical and analytical curves, especially in those predicting the position of the projectile. The ALE simulations predict a higher deceleration of the projectile, due to the fluid mesh that has been chosen to avoid the leakage problem. In general, it has been observed that the results by using ALE algorithm are very sensitive due to the Euler mesh $[21,33]$. Finally, it could be said that the numerical curves correlate quite well with the experimental ones showing the same trend.

\subsection{Cavity evolution}

An interesting parameter to analyze, that has been less studied, is the evolution of the cavity inside the fluid. Figs. 9 and 10 show a comparison of the cavity profile obtained both experimentally and numerically for different fluid-filled levels on three different instants at an impact velocity of $600 \mathrm{~m} / \mathrm{s}$. It can be seen that the size of cavity predicted by the simulations is very similar to the 


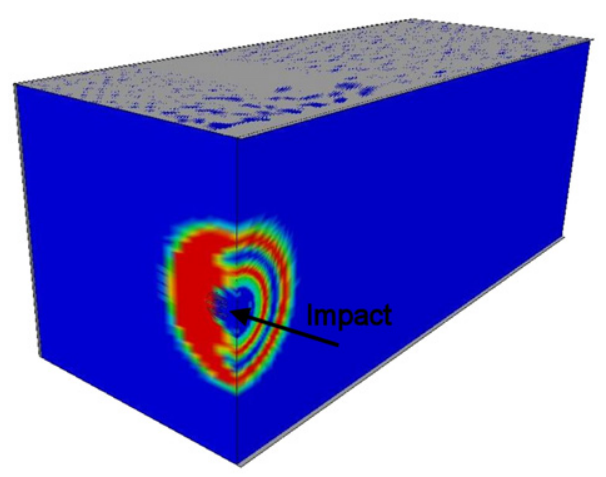

(a)

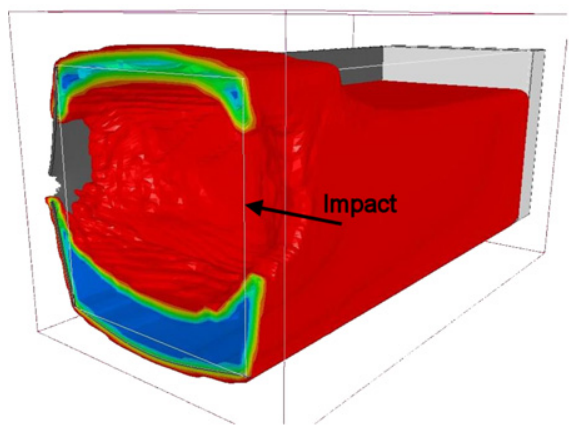

(c)

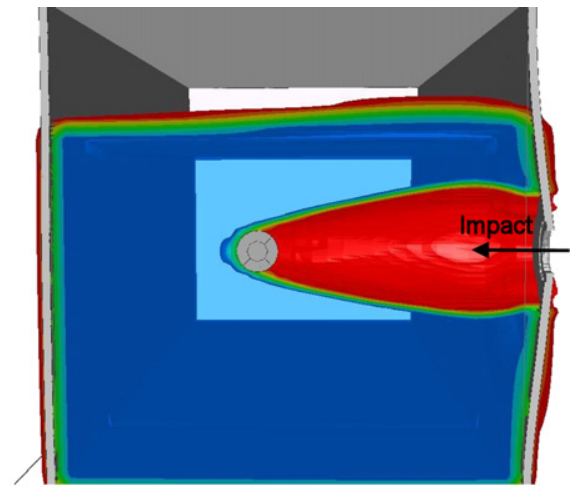

(b)

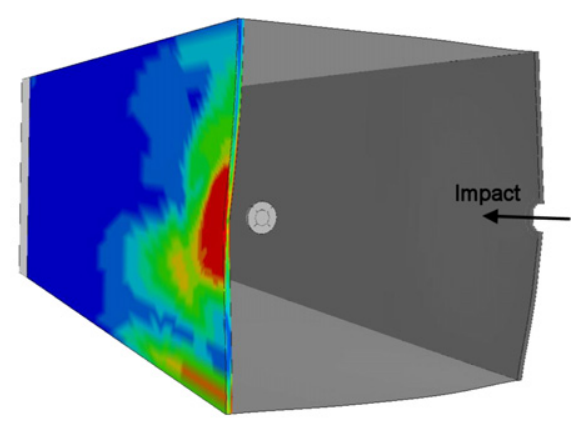

(d)

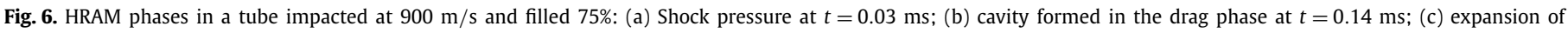
the cavity at $t=1.2 \mathrm{~ms}$; (d) contours of effective stress in the exit wall before being impacted at $t=0.25 \mathrm{~ms}$.

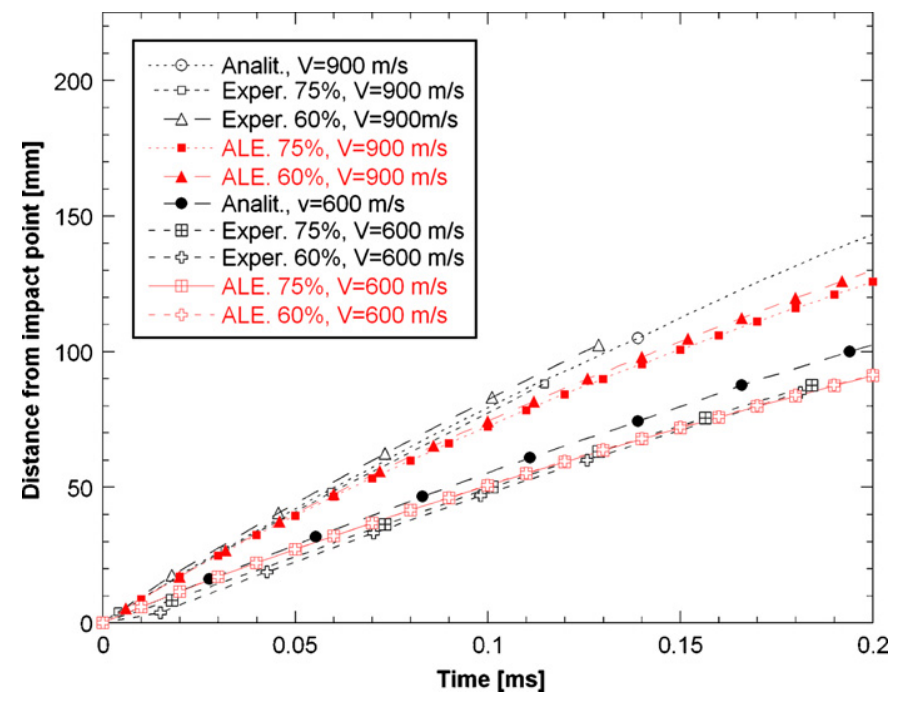

Fig. 7. Comparison of position of the projectile vs. time.

size observed in the experimental images; this is of great importance since the cavity is the main cause for the deformation of the walls. Special attention deserves the portion of fluid which is raised over the initial fluid level. This layer is the main responsible for the deformation of the upper wall.

\subsection{Pressure time history}

As was already mentioned before, two pressure gauges were used to record the time history of the HRAM pressure wave as it is propagated through the fluid. One of the pressure gauges (PTn)

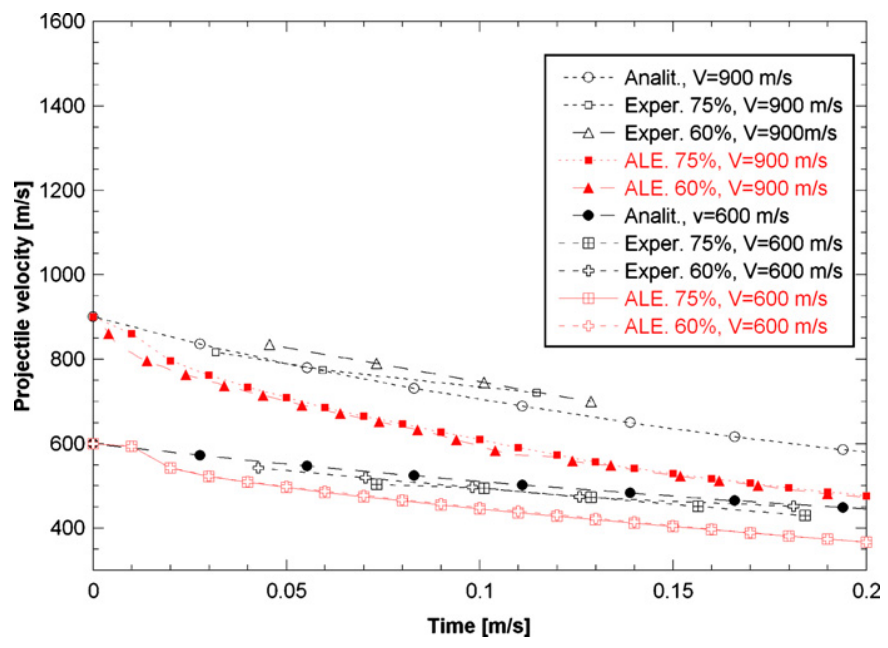

Fig. 8. Comparison of velocity decay vs. time.

was located near the impact point, at $30 \mathrm{~mm}$ from the wall and $75 \mathrm{~mm}$ from the shot line (Fig. 3), while the other (PTf) was in the middle of the tube, at $150 \mathrm{~mm}$ from the projectile trajectory (Fig. 3). The pressure time histories depicted in Figs. 11 and 12 show experimental and numerical curves representative of the pressure measurements at PTn and PTf at different velocities in a case of a tube filled at $75 \%$. The numerical curves correspond to the averaged value of several elements around each pressure gauge position. It can be seen that the pressure time history changes as a function of the location of the pressure gauge as well as the velocity of the projectile. It is observed that the maximum pressure value is not accurately predicted, specially on the PTf, nevertheless the trend of the influence of the different factors, position and 

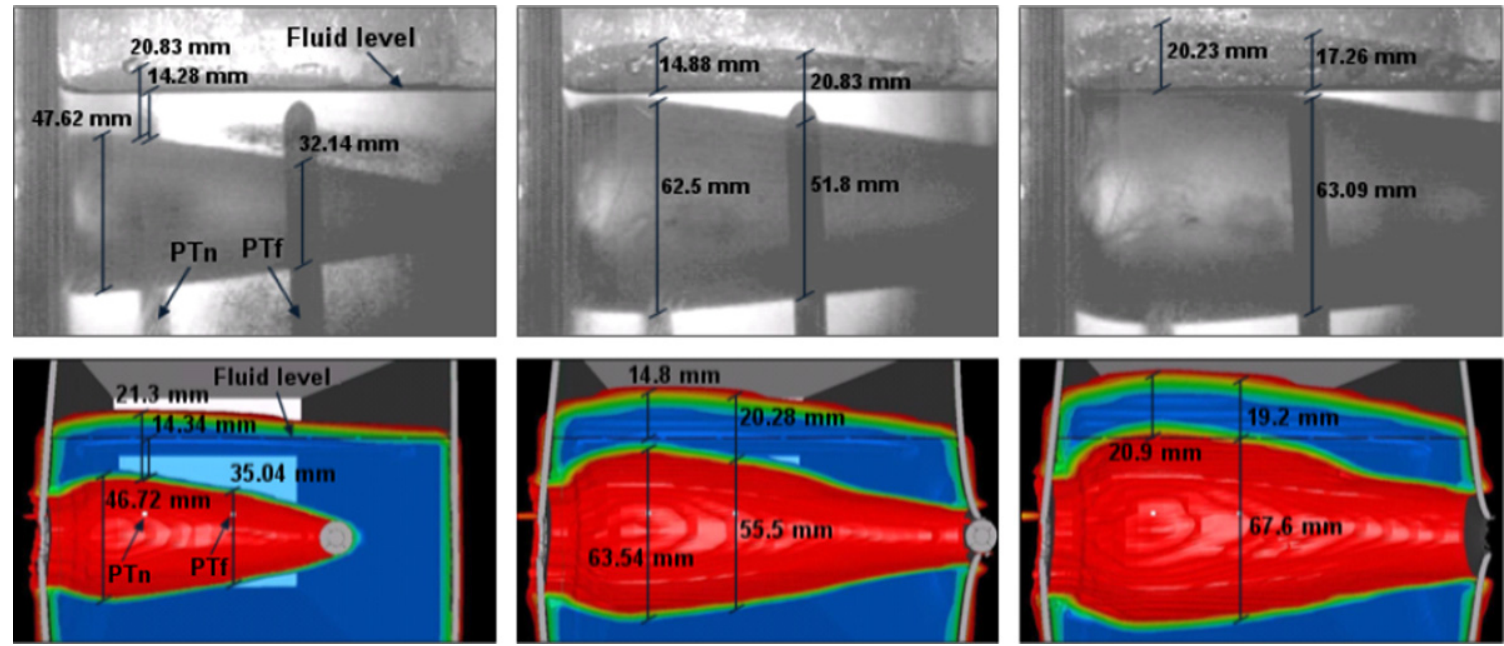

Fig. 9. Cavity evolution at $0.26,0.46$ and $0.60 \mathrm{~ms}$ obtained from experiments (top) and ALE simulation (bottom) for a tube impacted at $600 \mathrm{~m} / \mathrm{s}$, filled $75 \%$.
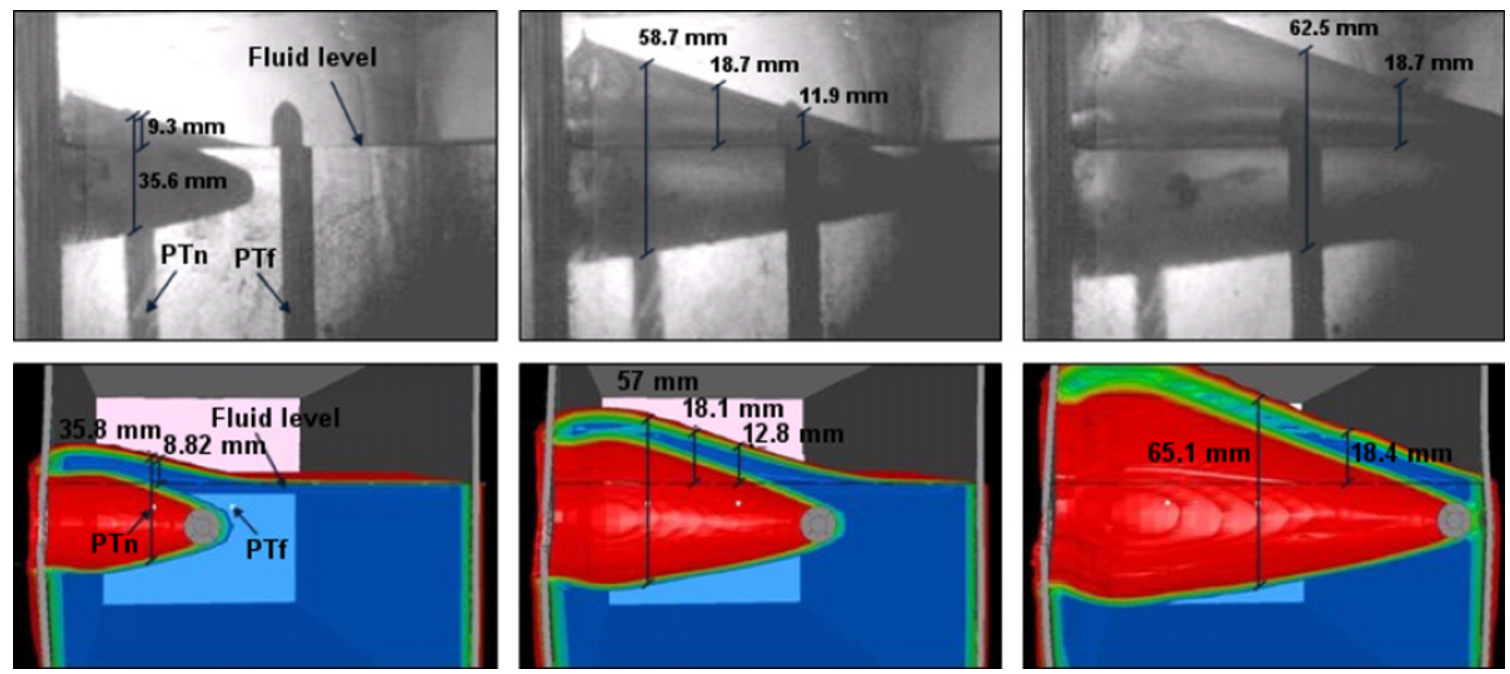

Fig. 10. Cavity evolution at $0.13,0.24$ and $0.38 \mathrm{~ms}$ obtained from experiments (top) and ALE simulation (bottom) for a tube impacted at $600 \mathrm{~m} / \mathrm{s}$, filled $60 \%$.

velocity, are well captured by the simulations. The beginning of the pressure pulse in the simulations coincides quite well with the experimental data, so the propagation of the pressure wave generated on the first stage of HRAM is well reproduced by the simulations.

The impulse, defined as the area $A_{p}$ below the time-pressure curve, was used by Wierzbicki and Moussa [47] to characterize the load applied by the fluid in an aircraft fuel tank under HRAM, uncoupling the fluid-structure interaction problem. Fig. 13 shows the impulse at each pressure transducer as a function of volume percentage for the different velocities considered. Data for the completely filled case has been added to the figure in order to have the complete information. It can be seen how the $A_{p}$ values obtained numerically and experimentally match very well. This is of great importance because the impulse, transmitted to the walls by the fluid, which has influence on the tube deformation, is well predicted. Furthermore, this figure confirms the capability of the numerical model for reproducing the influence of different parameters, as impact velocity, volume percentage and transducer position, on the fluid pressure.

\subsection{Deformations}

In this section, experimental deformation data in the entry and exit walls of the tubes are compared with the simulations results.

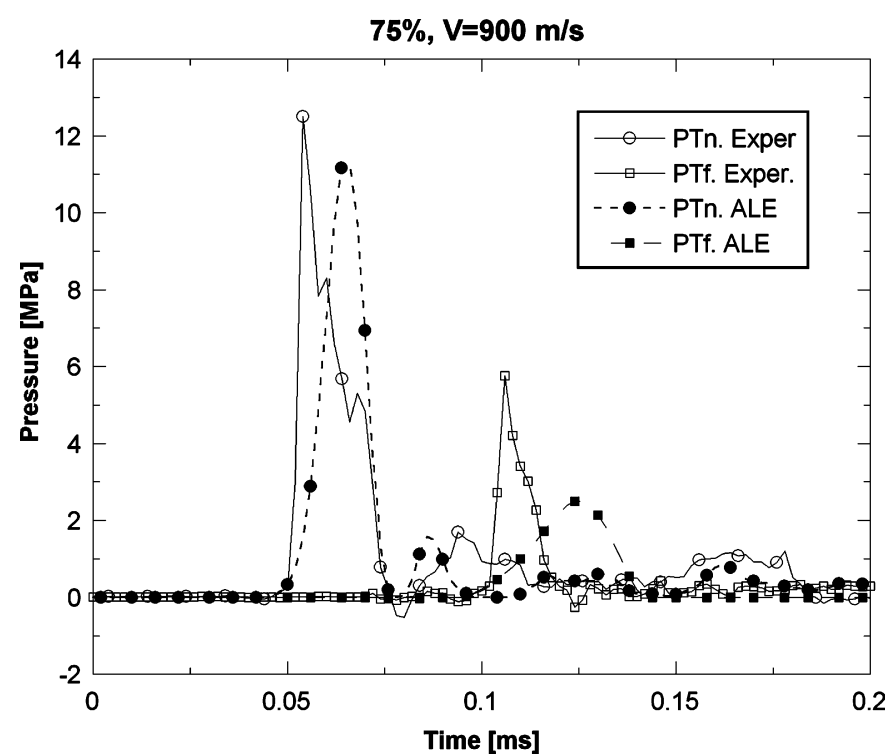

Fig. 11. Pressure time history near (PTn) and far (PTf) from the impact point in a tube $75 \%$ filled, impacted at $900 \mathrm{~m} / \mathrm{s}$. 


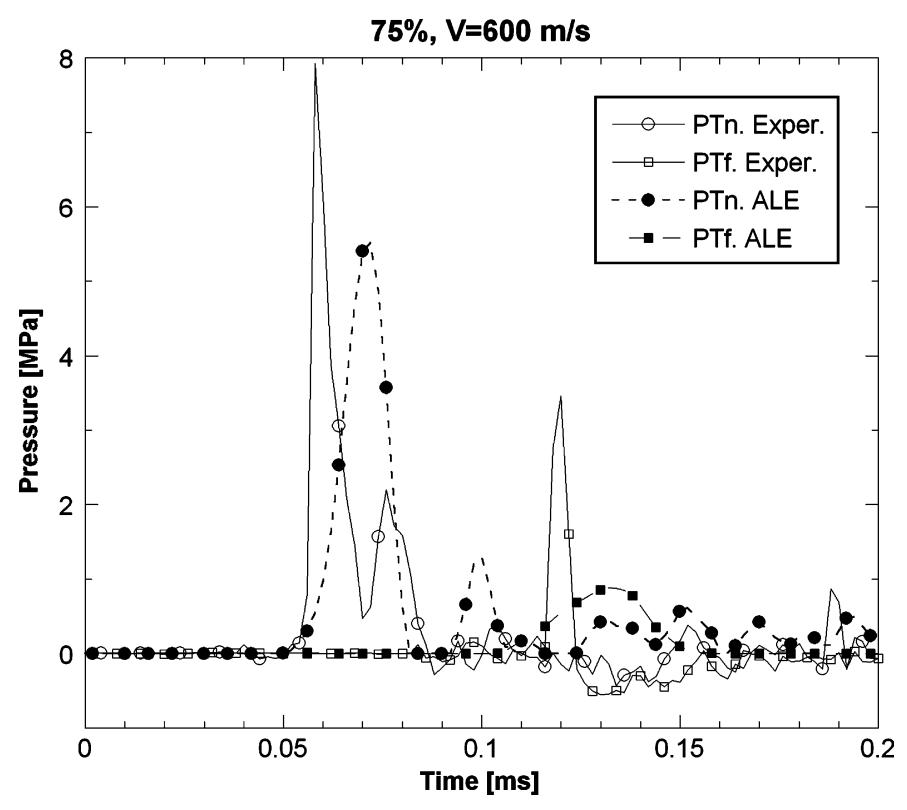

Fig. 12. Pressure time history near (PTn) and far (PTf) from the impact point in a tube $75 \%$ filled, impacted at $600 \mathrm{~m} / \mathrm{s}$.

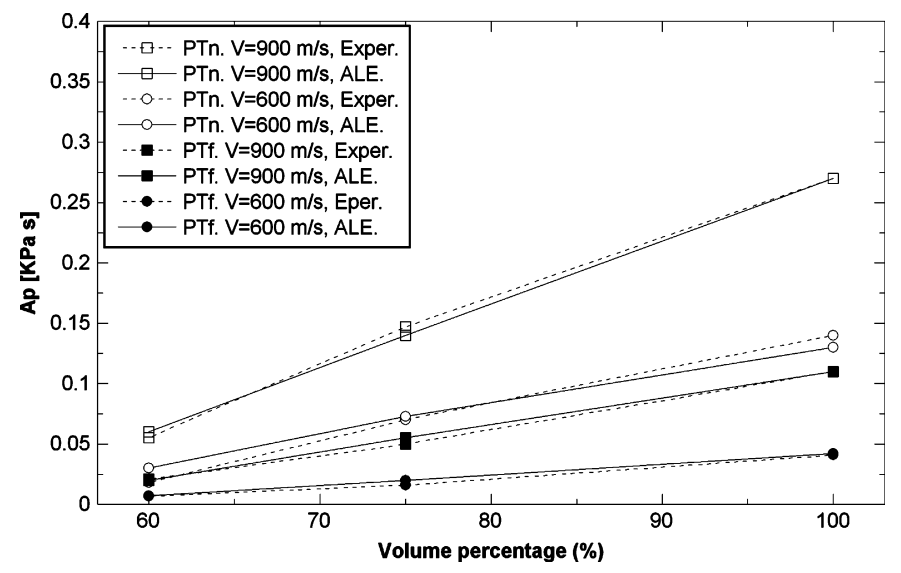

Fig. 13. Area below pressure/time curves vs. volume percentage.

Deformation of the walls is of interest due to its potential influence on the behaviour of contiguous cells that can be part of a whole fuel tank system. Figs. 14 and 15 depict the deformation of the entry and exit walls, all along the tube, for filling percentage of $75 \%$, impacted at 900 and $600 \mathrm{~m} / \mathrm{s}$. The time in the simulations was long enough to assure the stabilization of the displacements in the walls.

The figures show that the affected zone in which the deformation is produced is well defined in the simulations, allowing to know the limits of its extension. It is also observed that the deformation obtained in the simulations is smaller than that measured experimentally, nevertheless the trends of the walls response to the HRAM phenomenon are the same. The results of the deformation of the entry and exit walls for a tube filled at $60 \%$, impacted at 900 and $600 \mathrm{~m} / \mathrm{s}$ showed the same behaviour. Considering the mentioned observations, it can be said that the numerical model predicts in an appropriate way the influence of the considered factors, impact velocity and volume percentage, on the behaviour of partially filled tube walls. This is important to identify possible solutions in order to mitigate the influence of different factors.
$75 \%, V=900 \mathrm{~m} / \mathrm{s}$

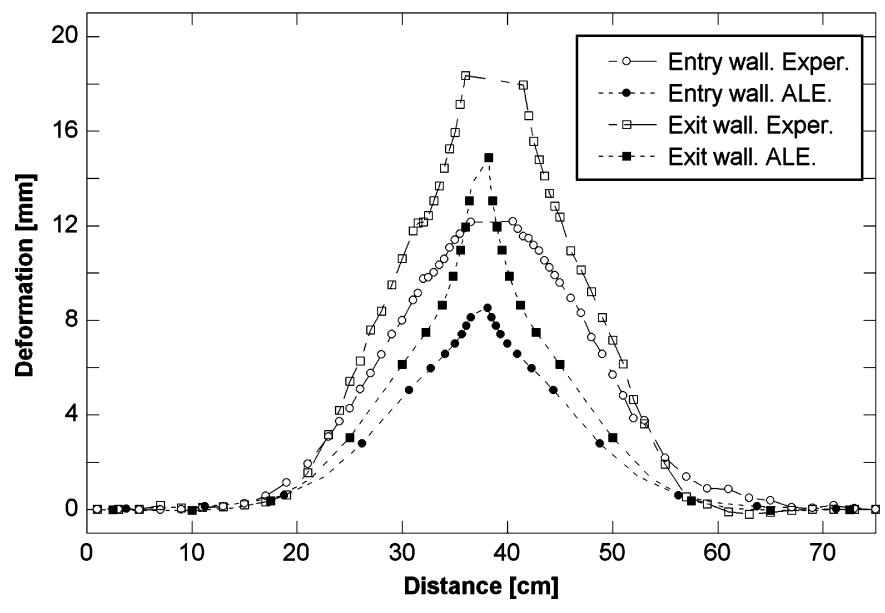

Fig. 14. Experimental and numerical deformation on the entry and exit wall of a tube filled $75 \%$, impacted at $900 \mathrm{~m} / \mathrm{s}$.

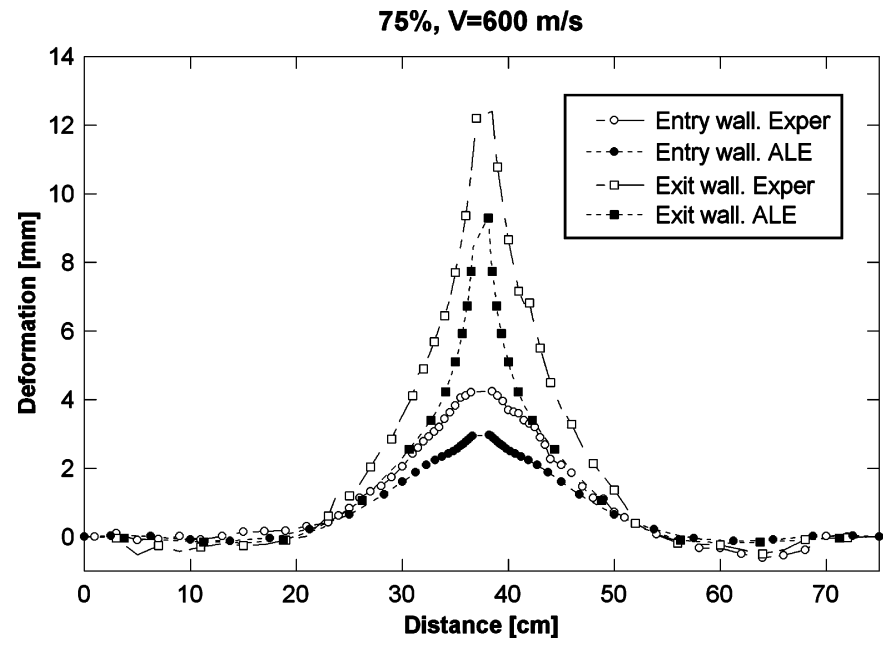

Fig. 15. Experimental and numerical deformation on the entry and exit wall of a tube filled $75 \%$, impacted at $600 \mathrm{~m} / \mathrm{s}$.

\section{Conclusions}

The capability of the ALE simulation approach in simulating the Hydrodynamic Ram phenomenon in partially filled tubes was studied in this work. Results of the numerical model were compared with experimental results in partially water-filled aluminium square tubes, impacted at different velocities.

The ALE technique is capable of faithfully reproducing the stages of the Hydrodynamic Ram in partially filled tubes: shock, drag, cavitation and exit phases from a qualitative and quantitative perspective. The cavity evolution, main cause of the tank final deformation, is accurately reproduced as compared with the images taken from the experimental tests. The projectile deceleration is also well reproduced for the different filling percentages; this fact is of great importance, since the kinetic energy loosed by the projectile is transferred to the structure (in the form of plastic deformation and damage) through the fluid.

The pressure, impulse and deformations obtained numerically have been compared with the experimental results showing that globally, the numerical model developed captures the influence of the different factors considered. This fact will allow to analyze in a more closely way the Hydrodynamic Ram phenomenon in order to try its attenuation in future designs, avoiding the expensive cost of full scale impact tests. 


\section{Acknowledgements}

The authors are indebted to the Spanish Ministry of Education (project DPI2008-06408/DPI), and to the Region of Madrid (project CCG08-UC3M/MAT-4464) for the financial support.

\section{References}

[1] M. Anghileri, L.-M.L. Castelleti, M. Tirelli, Fluid-structure interaction of water filled tanks during the impact with the ground, International Journal of Impact Engineering 31 (3) (2005) 235-254.

[2] R.E. Ball, Aircraft fuel tank vulnerability to hydraulic ram: modification of the Northrup finite element computer code BR-1 to include fluid-structure interaction. Theory and user's manual for BR-1HR, NPS-57B, July 1974, p. 74071.

[3] R.E. Ball, Structural response of fluid-containing-tanks to penetrating projectiles (hydraulic ram). A comparison of experimental and analytical results, NPS-57B, Naval Post Graduate School, Monterrey, California, 1976, p. 76051.

[4] R.E. Ball, A computer program for the geometrically nonlinear static and dynamic analysis of arbitrarily loaded shells of revolution. Theory and user's manual, NASA CR-1987.

[5] K.J. Bathe, H. Zhang, S. Ji, Finite element analysis of fluid flows fully coupled with structural interactions, Computers and Structures 72 (1999).

[6] S.J. Bless, P.F. Fry, J.P. Barber, Hydrodynamic ram driven pressure fields and panel displacements for high velocity spherical fragments, in: Proceedings of the Hydrodynamic Ram Seminar, May 1977, p. 37.

[7] T. Børvik, A.G. Hanssen, M. Langseth, L. Olovsson, Response of structures to planar blast loads-A finite element engineering approach, Computers and Structures 87 (2009) 507-520.

[8] R. Boyd, R. Royles, K.M.M. El-Deeb, Simulation and validation of UNDEX phenomena relating to axisymmetric structures, in: 6th International LS-DYNA Users Conference Simulation 2000, Dearborn, Michigan, April 9-11, 2000.

[9] E.L. Fasanella, R.L. Boinott, S. Kellas, Test and analysis correlation of high speed impacts of ice cylinders, in: 9th International LS-DYNA Users Conference, Dearborn, Michigan, June 4-6, 2006.

[10] C.J. Freitas, C.E. Anderson Jr., J.D. Walker, D.L. Littlefield, Hydrodynamic ram: A benchmark suite, in: Structures Under Extreme Loading Conditions, PVPvol. 325, ASME, New York, 1996, pp. 63-74.

[11] P.F. Fry, R.K. Newman, S.J. Bless, Measurements of hydrodynamic ram-driven panel displacements and comparison with numerical predictions by BR1A(HR) and Afton codes, in: Proceedings of the Hydrodynamic Ram Seminar, May 1977, p. 503.

[12] P.M. Gerhart, R.J. Gross, J.I. Hochstein, Fundamentals of Fluid Mechanics, Addison-Wesley Publishing Co., 1992.

[13] J.O. Hallquist, LS-DYNA Theoretical Manual, Livermore Software Technology Corporation, March 2006.

[14] A.G. Hanssen, Y. Girard, L. Olovsson, T. Berstad, M. Langseth, A numerical model for bird strike of aluminium foam-based sandwich panels, International Journal of Impact Engineering 32 (2006) 1127-1144.

[15] W.M. Herlin, J.G. Avery, Hydraulic ram structural response computer program (HRSR), Boeing Co., prepared under contract N60530-80-C-0242 for Naval Weapons Center, China Lake, California.

[16] W.W. Jarzab, R. Chwalinski, W.E. Pfrang, G. Tokar, Fluid-structure interaction effects in tank structures due to sloshing and hydrodynamic ram coupled Lagrangian-Eulerian simulations, in: Proc. International Conference: Spacecraft Structures and Mechanical Testing, Noordwijk, The Netherlands, 1988.

[17] S.T. Jenq, F.B. Hsiao, I.C. Lin, D.G. Zimcik, M. Nejad Ensan, Simulation of a rigid plate hit by a cylindrical hemi-spherical tip-ended soft impactor, Computational Materials Science 39 (2007) 518-526.

[18] G.R. Johnson, W.H. Cook, A constitutive model and data for metals subjected to large strains, high strain rates, and temperatures, in: Proceedings of 7 th Int. Symp., The Hague, The Netherlands, 1983, pp. 1-7.

[19] D. Karagiozova, N. Jones, Dynamic buckling of elastic-plastic square tubes under axial impact II: Structural response, International Journal of Impact Engineering 30 (2004) 167-192.

[20] O. Kayser-Herold, H.G. Matthies, A unified least-squares formulation for fluidstructure interaction problems, Computers and Structures 85 (11-14) (2007) 998-1011.

[21] J.H. Kim, H.C. Shin, Application of the ALE technique for underwater explosion analysis of a submarine liquefied oxygen tank, Ocean Engineering 35 (2008) 812-822.

[22] M. Koishi, T. Okano, L. Olovsson, H. Saito, M. Makino, Hydroplaning simulation using fluid-structure interaction in LS-DYNA, in: 9th International LS-DYNA Users Conference, Dearborn, Michigan, June 4-6, 2006.

[23] N. Lecysyn, A. Dandrieux, F. Heymes, L. Aprin, P. Slangen, L. Munier, C. Le Gallic, C. Dusserre, Ballistic impact on an industrial tank: Study and modeling of consequences, Journal of Hazardous Materials 172 (2009) 587-594.

[24] N. Lecysyn, A. Dandrieux, F. Heymes, P. Slangen, L. Munier, E. Lapebie, C. Le Gallic, C. Dusserre, Preliminary study of ballistic impact on an industrial tank:
Projectile velocity decay, Journal of Loss Prevention in the Process Industries 21 (2008) 627-634.

[25] K. Lee, H. Noguchi, S. Koshizuka, Fluid-shell structure interaction analysis by coupled particle and finite element method, Computers and Structures 85 (1114) (2007) 688-697.

[26] LS-DYNA Keyword User's Manual, Nonlinear Dynamic Analysis of Structures, Version 971, Livermore Software Technology Corporation, May 2007.

[27] Y. Lu, Z. Wang, Characterization of structural effects from above-ground explosion using coupled numerical simulation, Computers and Structures 84 (2006) 1729-1742.

[28] E.A. Lundstrom, Fluid/structure interaction in hydraulic ram, in: Proceedings of the Hydrodynamic Ram Seminar, May 1977, pp. 223-230.

[29] E.A. Lundstrom, Structural response of flat panels to hydraulic ram pressure loading, NWC TP 6770, Naval Weapons Center, 1988.

[30] P.O. Marklund, L. Nilsson, Simulation of airbag deployment using a coupled fluid-structure approach, in: 7th International LS-DYNA Users Conference, Dearborn, Michigan, May 19-21, 2002.

[31] M. Nishida, K. Tanaka, Experimental study of perforation and cracking of waterfilled aluminum tubes impacted by steel spheres, International Journal of Impact Engineering 32 (2006) 2000-2016.

[32] F. Petitpas, J. Massoni, R. Saurel, E. Lapebie, L. Munier, Diffuse interface mode for high speed cavitating underwater systems, International Journal of Multiphase Flow 35 (2009) 747-759.

[33] F. Poehlmann-Martins, J. Gabrys, M. Souli, Hydrodynamic ram analysis of nonexploding projectile impacting water, in: 2005 ASME Pressure Vessels and Piping Division Conference, Denver, Colorado, 17-21 July 2005.

[34] A. Rusinek, J.R. Klepaczko, Shear testing of a sheet steel at wide range of strain rates and a constitutive relation with strain-rate and temperature dependence of the flow stress, International Journal of Plasticity 17 (1) (Jan. 2001) 87-115.

[35] A. Rusinek, R. Zaera, J.R. Klepaczko, Constitutive relations in 3-D for a wide range of strain rates and temperatures. Application to mild steels, International Journal of Solids and Structures 44 (17) (Aug. 2007) 5611-5634.

[36] P. Santini, D. Palmieri, M. Marchetti, Numerical simulation of fluid-structure interaction in aircraft fuel tanks subjected to hydrodynamic ram penetration, in: 21st ICAS Congress, Melbourne, Australia, 1998.

[37] C.M. Seddon, K. Moodie, A.M. Thyer, M. Moatamedi, Preliminary analysis of fue tank impact, International Journal of Crash 9 (3) (2004) 237-244.

[38] M. Souli, L. Olovsson, I. Do, ALE and fluid-structure interaction capabilities in LS-DYNA, in: 7th International LS-DYNA Users Conference, Dearborn, Michigan, May 19-21, 2002.

[39] M. Souli, A. Ouahsine, L. Lewin, ALE formulation for fluid-structure interaction problems, Computer Methods in Applied Mechanics and Engineering 190 (2000) 659-675.

[40] C.E. Sparks, R.L. Hinrichsen, D. Friedmann, Comparison and validation of smooth particle hydrodynamic (SPH) and coupled Euler Lagrange (CEL) techniques for modelling hydrodynamic ram, in: 46th AIAA ASME/ASCE/AHS/ASC Structures, Structural Dynamics and Materials Conf., Austin, Texas, 18-21 April 2005.

[41] C.H. Tai, K.M. Liew, Y. Zhao, Numerical simulation of 3D fluid-structure interaction flow using an immersed object method with overlapping grids, Computers and Structures 85 (11-14) (2007) 749-762.

[42] R. Unger, M.C. Haupt, P. Horst, Application of Lagrange multipliers for coupled problems in fluid and structural interactions, Computers and Structures 85 (11-14) (2007) 796-809.

[43] D. Varas, J. López-Puente, R. Zaera, Experimental analysis of fluid filled aluminium tubes subjected to high velocity impact, International Journal of Impact Engineering 36 (2009) 81-91.

[44] D. Varas, R. Zaera, J. López-Puente, Numerical modeling of the hydrodynamic ram phenomenon, International Journal of Impact Engineering 36 (2009) 363374.

[45] M. Vesenjak, M. Matthaei, H. Mullerschon, Z. Ren, Fluid models in LS-DYNA and their interaction with a structure in dynamic simulations, in: Proceedings of PVP2005, ASME Pressure Vessels and Piping Division Conference, Denver 2005.

[46] J. Vierendels, L. Lanoye, J. Degroote, P. Verdonck, Implicit coupling of partitioned fluid-structure interaction problems with reduced order models, Computers and Structures 85 (11-14) (2007) 970-976.

[47] T. Wierzbicki, N.A. Moussa, Deformation and rupture of an aircraft fuel tank under hydraulic ram pressure loading, in: Proceedings of the 63rd Shock and Vibration Symposium, vol. 2, Las Cruces, New Mexico, 1992.

[48] C.C. Yang, Material properties of square tube, in: The 9th Fundamental Scientific Conference of Chinese Armed Forces Academy, Taiwan, ROC, vol. 1, 2002, pp. B1-B11.

[49] F.J. Zerilli, R.W. Armstrong, Dislocation-mechanics based constitutive relations for material dynamic calculations, Journal of Applied Physics 61 (5) (1987) 1816-1825.

[50] A. Zhang, K. Suzuki, A comparative study of numerical simulations for fluidstructure interaction of liquid-filled tank during ship collision, Ocean Engineering 34 (2007) 645-652. 